\title{
Transfer of siRNA against XIAP induces apoptosis and reduces tumor cells growth potential in human breast cancer in vitro and in vivo
}

\author{
Yingchao Zhang $\cdot$ Yu Wang $\cdot$ Wenxin Gao $\cdot$ \\ Ruhui Zhang $\cdot$ Xichun Han $\cdot$ Mingku Jia . \\ Wenzeng Guan
}

Published online: 15 February 2007

(C) Springer Science+Business Media B.V. 2007

\section{Erratum to: Breast Cancer Res Treat \\ DOI 10.1007/s10549-005-9080-0}

Unfortunately an incorrect Fig. 9 was published in the article. Below you will find the correct figure:

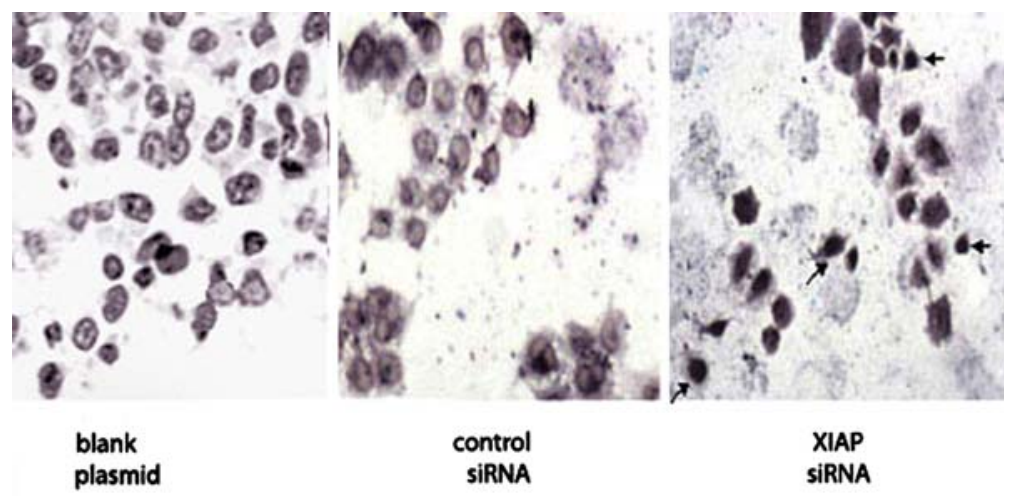

Fig. 9 siRNA to XIAP increases apoptosis in vivo. siRNA to XIAP or a scrambled nucleotide control siRNA or blank plasmid were injected into human breast cancer xenografts in nude mice as described in the Materials and methods section, and sections were obtained for analysis of XIAP expression and apoptosis. Representative adjacent sections are shown following (a) immunohistochemistry for XIAP expression, (b) TUNEL assay to detect apoptotic cells in situ. MCF-7 cells were grown on coverslips and

The online version of the original article can be found at http:// dx.doi.org/10.1007/s10549-005-9080-0

Y. Zhang · Y. Wang $(\bowtie) \cdot$ X. Han · M. Jia · W. Guan Department of Surgery, The Second Hospital of Jilin University, 218 Ziqiang Street, Changchun 130041, P.R. China

e-mail: wangyu720529@yahoo.com

W. Gao

Department of Oral Mucosal Disease, The Stomatology

Hospital of Jilin University, Changchun, P.R. China transfected with XIAP siRNA or control siRNA or blank plasmid. Cells were then analyzed for apoptosis with the TUNEL assay. Dark-blue staining of nuclei indicates apoptosis. Arrows indicate representative TUNEL-positive cells. (c) Quantitative presentation of the experiments in panel B. The percentages of TUNEL positive cells were scored for MCF-7 cells transfected with XIAP siRNA, control siRNA or blank plasmid.

\section{R. Zhang}

Department of Oral Surgery, The Stomatology Hospital of Jilin University, Changchun, P.R. China 